\title{
Die Geschichte des Ehrengerichts- und Disziplinarverfahrens der Wirtschaftstreuhänder
}

\author{
The History of the Court of Honour and Disciplinary Proceedings \\ against Chartered Public Accountants and Tax Consultants
}

The Chamber of Chartered Public Accountants and Tax Consultants was established in 1945 and issued its Regulations for Court of Honour and Disciplinary Proceedings, which contained substantial disciplinary provisions as well as procedural rules, in 1949. The substantial disciplinary provisions were for the first time regulated by state law in the Wirtschaftstreuhänder-Berufsordnung (1955), followed by the procedural rules in the Wirtschaftstreuhänderdisziplinarordnung (1962). A fundamental change took place in 1999 with the enactment of the Wirtschaftstreuhandberufsgesetz, which established type-based penal provisions and an enhanced liberalisation. With the introduction of the Administrative Courts on $1^{\text {st }}$ January 2014, the Disciplinary High Council as a specialised appeals authority was abolished; appeals against first-instance decisions are now referred to the Administrative Court for the respective province.

Keywords: Austrian chamber of chartered public accountants and tax consultants chartered accountants - disciplinary law - professional misconduct - tax consultants

\section{Die Entstehung des freien Berufs der Wirtschaftstreuhänder in Österreich}

Die Wirtschaftstreuhandberufe sind freie Berufe, verkündet $\S 1$ Abs. 2 WTBG. ${ }^{1}$ Das ist das Ergebnis eines langen Kampfes. Wurden die Tätigkeiten, die heute den Wirtschaftstreuhändern obliegen, ursprünglich in der ökonomisch eher unterentwickelten Monarchie hauptsächlich von Handelsschullehrern, Bankbeamten und Buchhaltern im Nebenberuf ausgeübt, ${ }^{2}$ war dann in der Ersten Republik die Rechtsgrundlage für die Berufsausübung ein Gewerbeschein, der keine andere Voraussetzung als die Anmel-

\footnotetext{
${ }^{1}$ BGB1. I 58/1999.

${ }^{2}$ NEUHAUSER, Österreichs Wirtschaftstreuhänder 10; SCHMIDT, Wirtschaftstreuhänderkammergesetz 1.
}

dung hatte. ${ }^{3}$ Das Gewerbe eines Buchsachverständigen und Bücherrevisors wurde als freies Gewerbe behandelt. Die Forderung des 1920 gegründeten Reichsverbandes der Bücherrevisoren nach einer Gleichstellung mit den freien Berufen blieb in der Ersten Republik unerfüllt. Im Jahr 1935 wurden mit der vom Bundesministerium für Handel und Verkehr auf Grund des Gewerbebund-Gesetzes BGBl. 84/1935 erlassenen Verordnung BGBl. 229/1935 die Buchsachverständigen, Bücherrevisoren, Finanz- und Wirtschaftsberater in einen Topf mit Pfandleihern, Kreditvermittlern, Theaterkartenbüros usw. geworfen und in der Innung der Beratungs- und Vermittlungsgewerbe zusammenge-

\footnotetext{
${ }^{3}$ NeUHAUSER, Österreichs Wirtschaftstreuhänder 13; SCHMIDT, Wirtschaftstreuhänderkammergesetz 3f.
} 
fasst. ${ }^{4}$ Erst der Anschluss an das Deutsche Reich im März 1938 brachte „bedeutsame Antriebe“ für die Entwicklung hin zum freien Beruf, ${ }^{5}$ weil damals die reichsdeutschen Rechtsvorschriften auf Österreich (die „Donau- und Alpen-Gaue“) übertragen wurden. Im Deutschen Reich war die Entwicklung weiter fortgeschritten. Schon 1931 war dort der Wirtschaftsprüferberuf im heutigen Sinn entstanden; in der Ländervereinbarung über die öffentliche Bestellung der Wirtschaftsprüfer ${ }^{6}$ schien erstmals die Bezeichnung „öffentlich bestellter Wirtschaftsprüfer" auf. Ab dem 15. Februar 1932 waren die Interessen dieses neuen Berufsstandes vom Institut der Wirtschaftsprüfer wahrgenommen worden. ${ }^{7}$ Nach dem 13. März 1938 wurden neben den handelsrechtlichen auch die steuerrechtlichen Vorschriften des Deutschen Reichs - wie die RAO vom 22. März $1931^{8}$ - übernommen. ${ }^{9}$ Der Wirtschaftsprüfer wurde gesetzlich verankert, indem mit der ersten und zweiten Verordnung zur Einführung handelsrechtlicher Vorschriften im Lande Österreich ${ }^{10}$ das deutsche Aktiengesetz aus 1937 in Österreich in Kraft gesetzt wurde. Außerdem wurde die oben erwähnte Ländervereinbarung in Österreich eingeführt. ${ }^{11}$ Bei den Handelskammern in Wien und Salzburg wurde je eine Zulassungs- und Prüfungsstelle für Wirt-

\footnotetext{
${ }^{4}$ SCHMIDT, Wirtschaftstreuhänderkammergesetz 4, WALDMANN, Entwicklung 7.

${ }^{5}$ LEIFER, Berufsrecht $1 \mathrm{f}$.

${ }^{6}$ dRGBl. 1931 I S. 761.

${ }^{7}$ NEUHAUSER, Österreichs Wirtschaftstreuhänder 16; Institut der Wirtschaftsprüfer, 75 Jahre WP 21f.

8 dRGBl. I, S 161.

${ }^{9} \mathrm{VO}$ zur Einführung steuerrechtlicher Vorschriften im Lande Österreich vom 14. 4. 1938 (dRGBl. I S. 389, GBlfLÖ. 94/1938) und vom 3. 8. 1938 (dRGBl. I S. 995); NEUHAUSER, Österreichs Wirtschaftstreuhänder 21.

${ }^{10}$ vom 11. 4.1938 (dRGBl. I S. 385, GBlfLÖ. 100/1938 und vom 2.8.1938 (dRGBl. I S.988 und GBIfLÖ. 389/1938); SCHMIDT, Wirtschaftstreuhänderkammergesetz 5.

${ }^{11}$ GBlfLÖ. 517/1938.
}

schaftsprüfer eingerichtet. ${ }^{12}$ Auch die Steuerberater und Helfer in Steuersachen erhielten mit §§ 107 und 107a RAO eine gesetzliche Grundlage. ${ }^{13}$ Der Reichsminister der Finanzen gründete 1943 die Reichskammer der Steuerberater; ${ }^{14}$ der Reichswirtschaftsminister gründete mit Verordnung vom 23. März 1943 die Reichskammer der Wirtschaftstreuhänder, in der die Wirtschaftsprüfer, Wirtschaftsprüfungsgesellschaften und die vereidigten Buchprüfer zusammengeschlossen waren. ${ }^{15}$

Dieses System, das zu einer Zersplitterung des Berufsstandes in zahlreiche Sparten führte, ${ }^{16}$ entsprach nicht den Interessen der österreichischen Wirtschaftstreuhänder, die eine alle verschiedenen Gruppen umfassende einheitliche Organisation - gleich den Ärzten, Rechtsanwälten, Notaren und dergleichen - angestrebt hatten. Mit dem Wiedererstehen der Republik Österreich im Jahr 1945 bot sich die Möglichkeit, alle einschlägig tätigen Personen in einer eigenen Kammer zusammenzufassen. Durch $\S 2$ des Verfassungsgesetzes vom 1. Mai 1945 über die Wiederherstellung des Rechtslebens in Österreich $^{17}$ wurden zunächst die reichsrechtlichen Bestimmungen auf dem Gebiet des wirtschaftlichen Prüfungs- und Treuhandwesens übernommen und blieben demnach in Kraft. ${ }^{18}$ Die Bezirksstellen der ehemaligen Reichskammern wurden durch einen Kommissar geleitet. ${ }^{19}$ Schon am 18. August 1945 ordnete das Staatsamt für Industrie, Gewerbe, Handel und Verkehr mit Erlass Zl. 20.219/IV/45 - unter gleichzeitiger Auflösung der bisher kommissarisch

\footnotetext{
${ }^{12}$ NeUHAUSER, Österreichs Wirtschaftstreuhänder 21; SCHMIDT, Wirtschaftstreuhänderkammergesetz 5.

${ }^{13}$ NEUHAUSER, Österreichs Wirtschaftstreuhänder 21.

${ }^{14}$ SCHMIDT, Wirtschaftstreuhänderkammergesetz 6.

${ }^{15}$ Institut der Wirtschaftsprüfer, 75 Jahre 42; SCHMIDT, Wirtschaftstreuhänderkammergesetz 6 .

${ }^{16}$ LEIFER, Berufsrecht 1.

17 StGBl. 6/1945.

18 SCHMIDT, Wirtschaftstreuhänderkammergesetz 7.

${ }^{19}$ SCHMIDT, Wirtschaftstreuhänderkammergesetz 8 .
} 
verwalteten Bezirksstelle Wien der Reichskammer der Wirtschaftstreuhänder - die provisorische Errichtung der Österreichischen Kammer der Wirtschaftstreuhänder an, deren Mitglieder die öffentlich bestellten Wirtschaftsprüfer und vereidigten Buchprüfer waren; über die Ausdehnung auf die gewerblich tätigen Buchsachverständigen und die Steuerberater sollte die Satzung Anordnungen treffen. ${ }^{20}$ Schließlich verabschiedete der Österreichische Nationalrat am 10. Dezember 1947 das WTKG, ${ }^{21}$ nach dessen $\S 1$ „zur Vertretung der gemeinsamen Interessen aller physischen und juristischen Personen sowie Personengemeinschaften, die innerhalb des Bundesgebietes zur Tätigkeit eines Wirtschaftstreuhänders ( $\S 3$ und 32) befugt sind", die Kammer der Wirtschaftstreuhänder als Körperschaft öffentlichen Rechtes errichtet wurde. §3 WTKG führte als Wirtschaftstreuhänder die Wirtschaftsprüfer, die vereidigten Buchprüfer und die Steuerberater an; §32 WTKG ordnete diesen Berufsgruppen Helfer in Steuersachen und Devisenberater sowie Buchsachverständige und ähnliche Sparten zu.

$\mathrm{Zu}$ einem freien Beruf gehört nach österreichischer Tradition eine eigene Disziplinargerichtsbarkeit, wie diese schon lange bei älteren freien Berufen wie Rechtsanwälten, Notaren und Ärzten eingerichtet war.

\section{Regelungen des Disziplinarverfahrens}

\subsection{Reichsdeutsche Vorschriften für Wirtschaftsprüfer}

Im Deutschen Reich gab es aufgrund der Satzung des Instituts der Wirtschaftsprüfer ein "Ehrenverfahren". Nach $§ 20$ dieses Statuts war ein Mitglied, das die Berufspflichten verletzte,

\footnotetext{
${ }^{20}$ SCHMIDT, Wirtschaftstreuhänderkammergesetz 9.

${ }^{21}$ BGBl. 20/1948.
}

gegen die Berufsehre verstieß oder sich innerhalb oder außerhalb der Ausübung des Berufs eines unwürdigen Verhaltens schuldig machte, ehrengerichtlich zu bestrafen. Es gab die Strafen der Verwarnung, des Verweises und einer Geldstrafe bis zu RM 1000,--. Nach § 21 waren Ehrengerichte $\mathrm{zu}$ bilden, die aus einem Obmann und zwei Beisitzern sowie deren Stellvertretern bestanden und aus dem Kreis der Mitglieder zu ernennen waren. Als zweite Instanz wirkte der Ehrengerichtshof, der aus einem Obmann und vier Beisitzern sowie deren Stellvertretern bestand. Ein Mitglied des jeweils amtierenden Ehrengerichtshofs musste die Befähigung zum Richteramt haben.

Als 1943 der Reichswirtschaftsminister mit Verordnung über den Zusammenschluss auf dem Gebiet des wirtschaftlichen Prüfungs- und Treuhandwesens ${ }^{22}$ die Reichskammer der Wirtschaftstreuhänder gründete, wurde sie damit Rechtsnachfolgerin des Instituts der Wirtschaftsprüfer, umfasste aber auch die vereidigten Buchprüfer. Nach $\S 20$ der Anordnung über die Reichskammer der Wirtschaftstreuhänder vom 30. März 1943 hatten die disziplinarrechtlichen und ehrengerichtlichen Vorschriften für Wirtschaftsprüfer weiter zu gelten. Die $\S \S 20$ bis 24 des Statuts wurden Bestandteil der Ehrengerichtsordnung für die Mitglieder der neu gegründeten Reichskammer. ${ }^{23}$

Durch einen an die Kammer für Handel, Gewerbe und Industrie gerichteten Erlass des Staatsamtes für Industrie, Gewerbe, Handel und Verkehr vom 26. Juni 1945 betreffend die erwerbsmäßige Tätigkeit auf dem Gebiet des wirtschaftlichen Prüfungs-, Beratungs- und Treuhandwesens wurde verfügt, dass bis zur Kundmachung eines Gesetzes über die Neugestaltung des Berufsrechtes die für die Zulassung, Prüfung, Bestellung und Vereidigung sowie für die

22 dRGBl. I Nr. 32 vom 30. 3. 1943.

${ }^{23}$ Vertrauliches Nachrichtenblatt der Reichskammer der Wirtschaftstreuhänder vom 30. 4. 1943. 
Ausübung der Berufstätigkeit von öffentlich bestellten Wirtschaftsprüfern, Wirtschaftsprüfungsgesellschaften und vereidigten Buchprüfern sowie für die Berufsaufsicht über diese Personen und das Disziplinarwesen geltenden Bestimmungen unter gewissenhafter Bedachtnahme auf das Recht der Republik Österreich und die allgemeinen volkswirtschaftlichen Ziele und Aufgaben des wirtschaftlichen PrüfungsBeratungs- und Treuhandwesens durch die in Betracht kommenden Dienststellen, Körperschaften und beteiligten Personen auch weiterhin sinngemäß anzuwenden und zu beachten sind. ${ }^{24}$ Zunächst waren daher in der Zweiten Republik die reichsdeutschen Disziplinarvorschriften für die in der ehemaligen Reichskammer der Wirtschaftstreuhänder vertretenen Personen anzuwenden.

\subsection{Schaffung eines einheitlichen Disziplinarrechts für alle Wirtschaftstreuhänder}

\subsubsection{Verordnung auf Grund formalgesetzlicher Delegation}

$\S 17$ Abs. 1 lit. c WTKG ordnete an, dass die Kammer verbindliche Vorschriften betreffend „Durchführung des Ehrengerichts- und Disziplinarverfahrens und Einrichtung des Ehrengerichts- und Disziplinarausschusses (EDO)“ festzusetzen habe. Nach § 17 Abs. 3 WTKG bedurfte diese Verordnung der Genehmigung des Bundesministeriums für Handel und Wiederaufbau. Eine nähere gesetzliche Determinierung fehlte. Auf Grund dieser formalgesetzlichen Delegation erließ die Kammer im Jahr 1949 mit Genehmigung des Ministeriums vom 1. Juni 1949 eine EDO, die am 14. Juni 1949 in Kraft trat. ${ }^{25}$ Die Verfasser des Entwurfs der Verordnung hatten sich - soweit es die Eigenheiten des Berufsstan-

\footnotetext{
${ }^{24}$ SCHMIDT, Wirtschaftstreuhänderkammergesetz 7.

${ }^{25}$ Amtsblatt der Kammer, Sondernr. 1/1949; HALPERN, Disziplinarwesen 30.
}

des der Wirtschaftstreuhänder zuließen - das seit Jahrzehnten bewährte Disziplinarstatut der Rechtsanwälte zum Vorbild genommen. ${ }^{26}$ In den folgenden Jahren wurde diese Verordnung einige Male abgeändert.

Die Verordnung sprach in $\S 1$ aus, dass der Vorstand der Kammer die unmittelbare Aufsicht über die in den $\S \S 3$ und 32 WTKG bezeichneten Personen ausübe (Abs. 1), dass die Befugnis des Präsidiums der Kammer zur Verhängung von Ordnungsstrafen unberührt bleibe (Abs. 2) und die oberste Aufsicht über die Handhabung der EDO dem Bundesministerium für Handel und Wiederaufbau im Einvernehmen mit dem Bundesministerium für Finanzen zustehe (Abs. 3).

Die näher bezeichneten Berufsgruppen von Wirtschaftstreuhändern unterlagen nach $\S 2$ der Verordnung ebenso wie die Berufsanwärter den Bestimmungen der EDO, wenn sie - wie $\S 3$ formulierte - die Pflichten ihres Berufs verletzt oder in- oder außerhalb des Berufes durch ihr Benehmen die Ehre oder das Ansehen des Standes beeinträchtigt hatten. $\S 4$ zählte demonstrativ solche Vergehen auf, wobei die Verletzung von Berufspflichten sowie das Ansehen schädigende Verhaltensweisen aufschienen. Als Disziplinarstrafen sah $\S 9$ die Verwarnung, den schriftlichen Verweis, die schriftliche strenge Verwarnung, Geldstrafen bis 5.000,-- Schilling sowie - überraschend - den Antrag an die zuständige Stelle auf ein befristetes Verbot der Berufsausübung oder auf dauernden Entzug der Berufsbefugnis vor. Dass gerade bei den schwersten Strafen dem erkennenden Senat nur die Antragstellung bei der zuständigen staatlichen Stelle eingeräumt wurde, ist damit zu erklären, dass die Kammer wegen der mangelnden gesetzlichen Grundlage der EDO befürchtete, der Verfassungsgerichtshof würde im Fall seiner Anrufung die Verordnung aufheben. ${ }^{27}$

\footnotetext{
${ }^{26}$ HALPERN, Disziplinarwesen 30.

${ }^{27}$ LEIFER, Berufsrecht 143.
} 
Nach $\S 5$ Abs. 1 EDO hatte „in den im $\S 3$ bzw. 4 angeführten Fällen der EDA einzuschreiten“. Dieser bestand aus dem Vorsitzenden, seinem Stellvertreter und einer bestimmten Anzahl von Beiräten und ebenso vielen Ersatzmännern, die aus dem Kreis der Kammermitglieder auf die Dauer von drei Jahren vom Vorstand der Kammer zu bestellen waren ( $\$ 5$ Abs. 2 EDO). Diese Bestellung konnte nur aus wichtigen Gründen abgelehnt werden ( $\$ 5$ Abs. 3 EDO). Der EDA übte seine Tätigkeit grundsätzlich in dreigliedrigen Senaten, bestehend aus einem Vorsitzenden und zwei Beiräten, aus. Bei der Zusammensetzung der Senate war darauf Bedacht zu nehmen, dass mindestens ein Mitglied der Berufsgruppe des Beschuldigten angehört ( $\$ 5$ Abs. 5 EDO).

Als Berufungs- und Beschwerdeinstanz war ein Berufungssenat einzurichten, der aus einem Vorsitzenden (seinem Stellvertreter), sowie zwei Beisitzern, alle aus dem Stand der Kammerangehörigen, sowie je einem Beamten aus dem Finanz- und dem Handelsministerium bestand (§6 Abs. 1 EDO). Auch dort musste mindestens ein Mitglied der Berufsgruppe des Beschuldigten angehören ( $₫ 6$ Abs. 3). Der Vorsitzende und sein Stellvertreter waren vom Bundesministerium für Handel und Wiederaufbau im Einvernehmen mit dem Bundesministerium für Finanzen auf Vorschlag des Vorstandes der Kammer zu bestellen (§ 6 Abs. 1 lit. a EDO).

Der Vorstand der Kammer hatte einen Kammeranwalt und dessen Stellvertreter zu bestellen. Diese mussten nicht Angehörige des Berufsstands der Wirtschaftstreuhänder sein (§ 16 Abs. 1 EDO). Tatsächlich hat die Kammer von Anfang an immer Angehörige der Justiz als Kammeranwälte bestellt. ${ }^{28}$

Die zentrale Figur des Verfahrens war der Vorsitzende des EDA, der sämtliche an den EDA gelangende Anzeigen zunächst einer Vorprü-

\footnotetext{
${ }^{28}$ HALPERN, Disziplinarwesen 31.
}

fung zu unterziehen hatte ( $\$ 17$ Abs. 1 EDO). Kam er in Übereinstimmung mit dem Kammeranwalt zur Überzeugung, dass schon nach dem Inhalt der Anzeige kein Tatbestand vorliege oder dass die Tat vor dem 27. April 1945 oder vor mehr als fünf Jahren begangen wurde, so konnte er die Anzeige dem zuständigen Senat mit dem Antrag auf Zurücklegung vorlegen (§ 17 Abs. 2 EDO). Sofern der Senat dem nicht folgte und die Einleitung des ordentlichen Verfahrens beschloss ( $\$ 17$ Abs. 3 EDO), hatte der Vorsitzende des EDA die Sache entweder an den Kosten- und Schlichtungsausschuss am Sitz der Kammer oder an einen Untersuchungsausschuss der örtlich zuständigen Landesstelle der Kammer zu leiten oder einen Untersuchungskommissär zu bestellen. Von der Einleitung des Verfahrens waren der Beschuldigte und etwaige Beschwerdeführer zu verständigen ( $§ 18$ Abs. 1 EDO).

Nach Vorliegen des Untersuchungsergebnisses konnte der Kammeranwalt beim Vorsitzenden eine Ergänzung begehren ( $\$ 18$ Abs. 3 EDO). Reichte jedoch das Untersuchungsergebnis für die Beurteilung aus, so hatte der Vorsitzende den Fall an den zuständigen Senat zu leiten, der nach Anhörung des Kammeranwalts den Beschluss zu fassen hatte, ob das Verfahren fortzusetzen oder einzustellen sei ( $\$ 18$ Abs. 4 EDO). Beschloss der Senat, dass kein hinreichender Grund zur Disziplinarbehandlung des Falls gegeben sei (Ablassungsbeschluss), so hatte der Vorsitzende hievon mit kurzer Begründung den Beschuldigten und gegebenenfalls auch den Beschwerdeführer schriftlich $\mathrm{zu}$ verständigen (§ 18 Abs. 5 EDO). Beschloss der Senat die „Einleitung“ - so die Diktion der Verordnung ( $\$ 18$ Abs. 6) -, hatte der Vorsitzende des jeweiligen Senats eine - nichtöffentliche ( $\$ 24$ Abs. 1 EDO) - mündliche Verhandlung auszuschreiben (§20 Abs. 1 EDO). Der Kammeranwalt hatte an jeder Verhandlung teilzunehmen ( $\$ 24$ Abs. 2 EDO).

Sofern der Beschuldigte der Verhandlung ohne Nachweis unabweislicher Verhinderungsgründe 
fernblieb und sich auch nicht vertreten ließ, war - bis zur Novelle $1955^{29}$ - das Disziplinarverfahren auf dieses ungerechtfertigte Fernbleiben auszudehnen und eine neuerliche Verhandlung auszuschreiben. Erschien der Beschuldigte auch $\mathrm{zu}$ dieser nicht, war das Verfahren in seiner Abwesenheit durchzuführen (§ 20 Abs. 1 EDO).

Vom Erkenntnis, das entweder sogleich zu verkünden oder der schriftlichen Ausfertigung vorzubehalten war, waren nicht nur der Beschuldigte, sondern auch der Vorstand der Kammer sowie das Handels- und das Finanzministerium zu verständigen ( $§ 29$ Abs. 1 EDO). Eine Berufung war innerhalb von 14 Tagen nach Zustellung des Erkenntnisses beim Vorsitzenden des EDA anzumelden ( $\$ 36$ Abs. 1 EDO) und innerhalb von weiteren 14 Tagen auszuführen ( $\$ 36$ Abs. 2 EDO). Die Beschwerde gegen die Kosten war innerhalb von 14 Tagen zu erheben ( $\$ 36$ Abs. 3 EDO).

Nach $\S 38$ EDO konnte der Vorsitzende des Berufungsausschusses die Verhandlungsakten an den Vorsitzenden des EDA zurückleiten und ihn ersuchen, von ihm bestimmt zu bezeichnende erforderliche Erhebungen zu veranlassen. Andernfalls hatte er den Berufungssenat einzuberufen und das Berufungsverfahren einzuleiten. Im Fall grober Verfahrensmängel war das Erkenntnis als nichtig aufzuheben und eine neuerliche Verhandlung erster Instanz anzuordnen. Das Gleiche galt, wenn sich das Verfahren erster Instanz als ergänzungsbedürftig erwies. Andernfalls war aufgrund der Aktenlage zu erkennen oder eine mündliche Verhandlung anzuordnen. Der Berufungssenat war nicht an die Anträge der Berufungswerber gebunden.

$\S 40$ EDO sah ein Strafregister für die Disziplinarstrafen vor. Drei Jahre nach Rechtskraft des Disziplinarerkenntnisses konnte um Löschung angesucht werden (Abs. 2).

\footnotetext{
${ }^{29}$ Amtsblatt der Kammer 2/1956.
}

Nach $\S 43$ Abs. 2 EDO waren auch die zur Zeit des Inkrafttretens der Verordnung bereits anhängigen Disziplinarfälle nach dieser Verordnung zu behandeln.

Mit der Novelle $1950^{30}$ wurde $\S 18$ Abs. 1 EDO dahin abgeändert, dass das ordentliche Verfahren in der Regel mit der Beschlussfassung des Vorsitzenden über den Antrag des Kammeranwalts zu beginnen habe, Vorerhebungen einzuleiten und einen Untersuchungskommissär zu bestellen. Es wurde auch an den Fall gedacht, dass nach der Sachlage keine Vorerhebungen notwendig sind. In diesem Falle konnte der Vorsitzende beschließen, den Fall unmittelbar dem zuständigen Senat zur Entscheidung über die Einleitung vorzulegen. In jedem Fall war der Beschuldigte davon zu verständigen.

\subsubsection{Gesetzliche Regelung des materiellen Disziplinarrechts}

1955 trat die Wirtschaftstreuhänderberufsordnung (WTBO) ${ }^{31}$ in Kraft, in welcher erstmals auf Ebene eines Gesetzes das materielle Disziplinarrecht geregelt wurde. Nach dessen $\S 47$ unterlagen alle Kammermitglieder einschließlich der Berufsanwärter der Ehrengerichtsbarkeit, wenn sie die Pflichten ihres Berufes verletzt oder inner- oder außerhalb des Berufs durch ihr Verhalten die Ehre oder das Ansehen des Standes beeinträchtigt haben. Als Strafen sah $\S 48$ die Verwarnung, die strenge Verwarnung, Geldbußen bis $10.000 \mathrm{~S}$ im Einzelfall, die Suspendierung bis zur Dauer eines Jahres oder die dauernde Entziehung der Berufsbefugnis vor. Gegen Berufsanwärter gab es als Strafe die Verkürzung der anrechenbaren Zeit sowie die Streichung als Berufsanwärter. Auch eine Verjährungsvorschrift wurde eingeführt: Nach $\S 50$ Abs. 1 WTBO verjährten die zu verfolgenden Handlungen in fünf Jahren ab dem Tag der Verfeh-

\footnotetext{
${ }^{30}$ Amtsblatt der Kammer, Sondernr. 3/1952.

${ }^{31}$ BGBl. 125/1955.
} 
lung oder dem Aufhören des verbotenen Verhaltens. Handelte es sich um eine Tat, die gegen Straf- oder Verwaltungsstrafgesetze verstieß, so endete die Verjährung nicht vor Eintritt der Strafverfolgung nach dem entsprechenden Gesetz.

Die EDO wurde daraufhin 1955 novelliert ${ }^{32}$ und in Übereinstimmung mit der WTBO gebracht, wobei deren materiellrechtliche Regelungen soweit nicht schon vorher vorhanden - nun gleichlautend aus dem Gesetz übernommen wurden, wie etwa in $\S 3$ die Verjährungsbestimmungen oder in $\S 9$ die Strafen. Nun war jede Anzeige dem Kammeranwalt zur Kenntnis zu bringen und dem Vorsitzenden zur Prüfung vorzulegen ( $\$ 17$ Abs. 1 EDO). In $\S 17$ Abs. 2 EDO wurde - im Einklang mit § 26 WTKG idF Novelle 1955 BGBl. 126/1955 - das Recht auf Verhängung von Ordnungsstrafen im Falle geringfügiger Verstöße dem Vorsitzenden des EDA eingeräumt.

\subsubsection{Gesetzliche Regelung des formellen Disziplinarrechts}

Erst mit Bundesgesetz vom 14. Februar 1962 über die Ehrengerichtsbarkeit für Wirtschaftstreuhänder und Berufsanwärter (Wirtschaftstreuhänder-Disziplinarordnung) ${ }^{33}$ wurde auch das Disziplinarverfahren durch ein eigenes Gesetz geregelt, worauf § 17 a WTKG idF der Novelle BGBl. 301/1984 verwies. Nach §1 Abs. 1 WTDO oblag die disziplinäre Überwachung der Berufsangehörigen und Berufsanwärter in erster Instanz dem EDA und in zweiter Instanz dem Berufungssenat. Der EDA hatte seine Tätigkeit in dreigliedrigen Senaten auszuüben; für den Bereich der Landesstellen Wien, Niederösterreich und das Burgenland waren drei Senate am Sitz der Kammer in Wien, im Übrigen je ein Senat bei jeder Landesstelle einzurichten $(\S 1$

\footnotetext{
${ }^{32}$ Amtsblatt der Kammer 2/1956.

${ }^{33}$ BGB1. 63/1962.
}

Abs. 2 WTDO). Der Berufungssenat bestand aus einem rechtskundigen Vorsitzenden und vier Beisitzern ( 2 Abs. 2 WTDO). Alle Senatsmitglieder waren vom Vorstand aus dem Stand der Wirtschaftstreuhänder zu bestellen ( $\$ 2$ Abs. 1 und 2 WTDO).

Der Vorsitzende des EDA verlor seine zentrale Stellung. Nach § 5 Abs. 1 WTDO oblag es ihm, die zur ordnungsgemäßen Geschäftsführung des EDA erforderlichen Verfügungen zu treffen und ihn nach außen zu vertreten. Allerdings behielt er das Recht, bei geringfügigen Verstößen mit einer Ordnungsstrafe vorzugehen, gegen die es einen Einspruch gab, der zur Einleitung des ordentlichen Verfahrens führte $(\S 1$ Abs. 6 WTDO). Er hatte weiterhin alle Anzeigen - jedoch ohne inhaltliche Prüfung - dem Beschuldigten zur Äußerung zuzustellen ( $\$ 7$ Abs. 1 WTDO). Danach begann die Tätigkeit des Kammeranwalts. Dieser hatte nach Prüfung der Anzeige an den zuständigen Senat entweder den Antrag auf Nichteinleitung oder auf Einleitung des ehrengerichtlichen Verfahrens zu stellen ( 8 Abs. 1 WTDO). Im Falle der Einleitung hatte der Vorsitzende des EDA einen Untersuchungskommissär $\mathrm{zu}$ bestellen $(\S 9$ Abs. 1 WTDO). Nach dem Ergebnis der Untersuchung hatte der Kammeranwalt an den Senat den Antrag auf Einstellung oder auf Verweisung zur mündlichen Verhandlung zu stellen ( 12 Abs. 1 WTDO). Die Beschlüsse über die Anträge des Kammeranwalts waren in nichtöffentlicher Sitzung zu fassen ( $\$ 12$ Abs. 2 WTDO). Im Falle eines Verweisungsbeschlusses hatte der Vorsitzende des Senats eine - nicht öffentliche ( $\$ 13$ Abs. 2 WTDO) - mündliche Verhandlung auszuschreiben ( $\$ 13$ Abs. 1 WTDO), nach der er das Erkenntnis immer sogleich zu verkünden hatte ( $\$ 16$ Abs. 1). Dagegen stand dem Beschuldigten und dem Kammeranwalt die Berufung zu (§ 17 Abs. 1 WTDO), die binnen zwei Wochen nach Zustellung des Erkenntnisses beim Vorsitzenden des EDA anzubringen war $(\S 17$ 
Abs. 2) und aufschiebende Wirkung hatte ( $§ 17$ Abs. 3).

Nach $\S 5$ Abs. 5 WTDO stand dem BM für Handel und Wiederaufbau die Aufsicht über die Handhabung des ehrengerichtlichen Verfahrens zu; es konnte jederzeit Berichte über die Tätigkeit des EDA einholen, Akteneinsicht nehmen und gesetzwidrige Beschlüsse und Verfügungen, soweit sie nur das Verfahren betrafen, aufheben. Zur Wahrung dieser Dienstaufsicht hatte es zu den Sitzungen des Berufungssenats einen Vertreter zu entsenden, der rechtzeitig zu verständigen war (§ 2 Abs. 2 letzter Satz WTDO). Diese Bestimmungen hob der Verfassungsgerichtshof als verfassungswidrig auf, weil der Berufungssenat im Hinblick auf die Strafbefugnisse ein unabhängiges und unparteiisches, auf Gesetz beruhendes Gericht (Tribunal) iSd Art 6 EMRK sein muss. ${ }^{34}$ Damit war die letzte mögliche Einflussnahme der staatlichen Verwaltung auf die Standesgerichtsbarkeit beseitigt.

\subsection{Tiefgreifende Umstellung des Disziplinarrechts im Jahr 1999}

Eine grundlegende Änderung des Disziplinarrechtes erfolgte durch das Bundesgesetz über die Wirtschaftstreuhandberufe (WTBG), ${ }^{35}$ das mit 1. Juli 1999 in Kraft getreten ist (§ 227 Abs. 1 leg. cit.). Dieses brachte ziemlich genau 50 Jahre nach Inkrafttreten der EDO das Ende der Ehrengerichtsbarkeit. Verfolgt werden nur noch Berufsvergehen, nicht mehr außerberufliches standeswidriges Verhalten. Folgerichtig trat an die Stelle des EDA der Disziplinarrat ( $\$ 122$ WTBG), an die Stelle des Berufungssenats der Disziplinaroberrat (§ 123 WTBG).

Außerdem brachte das WTBG eine wesentliche Liberalisierung und Entkriminalisierung:

\footnotetext{
${ }^{34}$ VfGH 11. 10. 1988, G 238/87-8 = VfSlg 11.872; PAUER, KLEMENZ, Befugnisse der Wirtschaftstreuhänder 471f. 35 BGBl. I 58/1999.
}

a) An die Stelle der Generalklausel des $\S 47$ WTBO trat ein Typenstrafrecht. Ausschließlich die in $\S 120$ WTBG aufgezählten Handlungen können nun zu einer Bestrafung im Rahmen des Disziplinarverfahrens führen. Ein großer Teil der Sachverhalte, auf Grund derer bisher die Verletzung des Standesansehens der Wirtschaftstreuhänder angenommen wurde, ist im Katalog des §120 WTBG nicht enthalten. Nicht einmal alle Verstöße gegen Berufspflichten bilden nunmehr ein Berufsvergehen. Am auffallendsten ist vielleicht, dass die Verletzung der Verschwiegenheitspflicht nach §91 WTBG im Katalog des $\S 120$ WTBG nicht aufscheint. Das war kein Redaktionsversehen, sondern geschah bewusst, behandelt doch § 116 WTBG die Verletzung der Verschwiegenheitspflicht als Verwaltungsübertretung. $\S 120 \mathrm{Z} 25$ WTBG sieht freilich als Berufsvergehen die Verletzung auch solcher Pflichten vor, die die Kammer in einer nach $\S 83$ WTBG zu erlassenden Richtlinie für die Ausübung der Wirtschaftstreuhandberufe festlegt. Durch diese Ausübungsrichtlinie, die 2003 erlassen wurde (WT-ARL 2003) ${ }^{36}$, hat sich die Zahl der Berufsvergehen und damit auch der Disziplinarverfahren entscheidend erhöht. Jetzt ist auch die Verletzung der Verschwiegenheitspflicht - wie der Verfassungsgerichtshof klargestellt hat ${ }^{37}$ - wieder disziplinär zu ahnden ( 1 WT-ARL 2003). Außerdem sieht die WTARL 2003 etwa eine näher umrissene Fortbildungsverpflichtung (§3), eine Krankenversicherungspflicht (§6), die Verpflichtung, bei Beendigung eines Auftragsverhältnisses dem Auftraggeber die von diesem übergebenen Unterlagen herauszugeben ( $\$ 9$ Abs. 3), die Pflicht zum korrekten Verhalten gegenüber Behörden ( $§ 10)$ sowie das Verbot des Missbrauchs von FinanzOnline (§ 11) vor.

\footnotetext{
${ }^{36}$ Amtsblatt der Kammer, Sondernr. II/2003.

37 VfGH 20. 2. 2014, B 1614/2013-7.
} 
b) Die Strafbefugnis der Disziplinarsenate wurde stark beschränkt. Nach §119 Abs. 1 WTBG gibt es nur noch die Strafe der Verwarnung oder eine Geldbuße in der Höhe von EUR 7.268,--; bei Verursachung eines schweren Schadens erhöht sich die Geldbuße bis auf das Doppelte.

c) $\$ 144$ WTBG rezipiert für das Disziplinarverfahren (u.a.) Vorschriften des Verwaltungsstrafgesetzes, darunter auch $\S 31$ VStG, der eine Verjährungsfrist von sechs Monaten ab dem $\mathrm{Ab}$ schluss der strafbaren Handlung oder dem Aufhören des strafbaren Verhaltens vorsah. (Mittlerweile wurde diese Frist mit BGBl. I 33/2013 auf ein Jahr verlängert).

Auch die Verfahrensbestimmungen wurden teilweise geändert:

Der Vorsitzende des EDA hat nur noch repräsentative Aufgaben, allerdings weiterhin das Recht auf Verhängung von Ordnungsstrafen in geringfügigen Fällen (§ 131 Abs. 4 WTBG).

Die Bedeutung des Kammeranwalts ist gestiegen. Er kann dann, wenn er nach Prüfung der Anzeige keine Gründe für die Weiterverfolgung findet, die Anzeige zurücklegen (§131 Abs. 1 WTBG). Andernfalls hat er die Anzeige dem Angezeigten mit der Aufforderung zuzustellen, binnen 14 Tagen Stellung zu nehmen ( $\$ 131$ Abs. 2 WTBG). Danach kann er die Anzeige zurücklegen (§ 132 Abs. 1 WTBG).

Wird die Anzeige nicht zurückgelegt, hat der Kammeranwalt an den zuständigen Senat den Antrag auf Einleitung eines Disziplinarverfahrens zu stellen. Auch dieser Antrag ist dem Angezeigten ehestens zur Kenntnis zu bringen; dieser hat dann Gelegenheit, binnen zwei Wochen eine Gegenäußerung abzugeben und einen Gegenantrag zu stellen. Von der Zurücklegung der Anzeige hat der Kammeranwalt den Angezeigten umgehend zu verständigen ( $\S 132$ Abs. 1 WTBG). Nach § 131 Abs. 1 WTBG hat er auch den Anzeiger von jeder Zurücklegung zu verständigen.
Die Regeln über den Untersuchungskommissär und dessen Vorgangsweise sowie über die mündliche Verhandlung blieben im Wesentlichen gleich. Die Verhandlung ist nun allerdings öffentlich (§136 Abs. 2 WTBG). Der Senat besteht aus vier Mitgliedern, die - wie bisher Standesangehörige sind (§ 124 Abs. 1 WTBG). Er hat mit Stimmenmehrheit seine Erkenntnisse zu fällen und seine Beschlüsse $\mathrm{zu}$ fassen. Im Fall der Stimmengleichheit entscheidet die Stimme des Vorsitzenden (§ 137 Abs. 1 WTBG).

Gegen das Erkenntnis war die Berufung an den Disziplinaroberrat binnen einer Frist von zwei Monaten vorgesehen, der so zusammengesetzt war, wie es die WTBO vorgesehen hatte ( $§ 140$ WTBG idF vor BGBl. I 121/2013). Gegen das Erkenntnis des Berufungssenates gab es nach wie vor kein ordentliches Rechtsmittel, allerdings eine Beschwerde an den Verwaltungsgerichtshof.

\subsection{Verwaltungsgerichts- barkeitsnovelle 2012}

Eine weitere einschneidende Änderung trat durch die Verwaltungsgerichtsbarkeitsnovelle (VwGNov) 201238 ein, die mit 1. Jänner 2014 in Kraft getreten ist. In deren Gefolge wurde mit BGBl. I 121/2013 der Disziplinaroberrat abgeschafft. Nunmehr gibt es gegen die Erkenntnisse des Disziplinarrats die Bescheidbeschwerde ( $§ 7$ $\mathrm{VwGVG}^{39}$ ) an das jeweils zuständige Landesverwaltungsgericht (Art. 131 Abs. 1 B-VG idF BGBl. I 164/2013), die binnen vier Wochen zu erheben ist ( $\$ 7$ Abs 4 VwGVG). Gegen Verfahrensanordnungen ist keine abgesonderte Beschwerde zulässig ( 7 Abs. 1 VwGVG).

Während also bis Ende 2013 sowohl in erster als auch in zweiter Instanz ausschließlich Mitglieder der Kammer der Wirtschaftstreuhänder tätig waren, endet diese Autonomie nun in der ersten

\footnotetext{
${ }^{38}$ BGBl. I 51/2012.

${ }^{39}$ BGBl. I 33/2013.
} 
Instanz. Im Unterschied zu den Disziplinarordnungen anderer freier Berufe wie jenen der Ärzte, der Ziviltechniker, der Apotheker, die die Beteiligung von hauptberuflich als Richter Tätigen an den Senaten vorsehen, lag bei den Wirtschaftstreuhändern die Ausübung der Gerichtsbarkeit allein in den Händen von Berufsangehörigen, also richterlichen Laien. Diese haben ihre Aufgabe mit großem Eifer, gewissenhaft und umsichtig erfüllt. Bei der Verhandlungsführung, noch mehr bei der Ausfertigung der Erkenntnisse - auch jener der zweiten Instanz - war aber oftmals die fehlende richterliche Routine $\mathrm{zu}$ erkennen. Selbstverständlich waren daher Beschwerden an den Verwaltungsgerichtshof - aus formalen Gründen - häufig erfolgreich.

Erfahrungen mit den Landesverwaltungsgerichten sind spärlich. Es gibt bis jetz ${ }^{40}$ noch keine einzige inhaltliche Entscheidung eines solchen Gerichtes, wohl aber in einer Beschwerdesache einen heftigen Kompetenzkonflikt zwischen den Landesverwaltungsgerichten von Wien und Niederösterreich. Zur Entscheidung des Kompetenzkonfliktes wurde der Verwaltungsgerichtshof angerufen, der noch nicht entschieden hat. Vor einem Landesverwaltungsgericht fand Anfang Dezember 2015 eine Verhandlung statt, die geschlossen wurde; die Entscheidung wurde der schriftlichen Ausfertigung vorbehalten (§ 29 Abs. 3 Z. 2 VwGVG).

Die Zukunft wird hoffentlich zeigen, dass sich die neue Verwaltungsgerichtsbarkeit in Disziplinarverfahren der Wirtschaftstreuhänder positiv auswirkt. Zweifellos ist die grundsätzliche Neugestaltung der Anfechtbarkeit von Verwaltungsentscheidungen zu begrüßen und bedeutet für den Rechtsstaat einen Fortschritt. Allerdings wissen wir von Nestroy: „Überhaupt hat der Fortschritt das an sich, dass er viel größer ausschaut, als er wirklich ist.“ („Der Schützling“ 4. Akt 10. Szene).

${ }^{40}$ Stand 4. 2. 2016.

\section{Korrespondenz:}

Dr. Erich Kodek

Senatspräsident des OGH i.R

Diesterweggasse 23,

1140 Wien

erichkodek@gmx.at

\section{Abkürzungen:}

EDA Ehrengerichtlicher Disziplinarausschuss

EDO Ehrengerichts- und Disziplinarordnung

GBlfLÖ. Gesetzblatt für das Land Österreich

RAO Reichsabgabenordnung

VwGVG Verwaltungsgerichtsverfahrensgesetz

WTBG Wirtschaftstreuhandberufsgesetz

WTKG Wirtschaftstreuhänder-Kammergesetz

Siehe auch das allgemeine Abkürzungsverzeichnis: [http://www.rechtsgeschichte.at/files/abk.pdf]

\section{Literatur:}

Romuald BERTL, Dieter MANDL, Gerwald MANDL, (Hgg.), Handbuch für Wirtschaftstreuhänder. Die Praxis des Steuerberaters, Buchprüfers und Wirtschaftsprüfers (Wien 1989).

Robert HALPERN, Das Disziplinarwesen der Kammer, in: Amtsblatt der Kammer der Wirtschaftstreuhänder, Sondernummer anlässlich des Kammertages am 10. Dezember 1957 „10 Jahre Wirtschaftstreuhänder-Kammergesetz".

Institut der Wirtschaftsprüfer (Hg.), 75 Jahre Wirtschaftsprüfer im IDW. Rückblicke (Düsseldorf 2007).

Franz LEIFER, Das Berufsrecht der Wirtschaftstreuhänder (Wien 1957).

Thomas NeUHAUSER, Österreichs Wirtschaftstreuhänder. Die Geschichte der Kammer der Wirtschaftstreuhänder (o.J.)

Dietmar PAuer, Claudia Klemenz, Befugnisse der Wirtschaftstreuhänder - Abgrenzung zu anderen (freiberuflichen) Tätigkeiten, in: BERTL, MANDL, MANDL, Handbuch $457 \mathrm{ff}$.

Kurt SCHMIDT (Hg.), Das Wirtschaftstreuhänderkammergesetz (Wien 1948).

Ludwig WALDMANN, Die historische Entwicklung des Berufsstandes, in Festschrift 25 Jahre Kammer der Wirtschaftstreuhänder (Wien 1973). 